\title{
Análise de conteúdo estomacal de espécies de Pipridae e Thraupidae (Aves, Passeriformes) da Mata Atlântica do Baixo Sul da Bahia, Brasil
}

\author{
Rozivane de Jesus Silva ${ }^{1}$ e Caio Graco Machado ${ }^{2}$ \\ 1. Bolsista PROBIC/UEFS, Graduando em Ciências Biológicas, Universidade Estadual de Feira de Santana, e-mail: \\ rozivanee@hotmail.com \\ 2. Orientador, Departamento de Ciências Biológicas, Universidade Estadual de Feira de Santana, e-mail: gracom@uol.com.br
}

PALAVRAS-CHAVE: Dieta, conteúdo estomacal, grupo trófico.

\section{INTRODUÇÃO}

O Brasil apresenta uma das maiores diversidades de aves do mundo, com 1901 espécies, das quais 270 espécies endêmicas do Brasil (Comitê Brasileiro de Registros Ornitológicos 2014). A avifauna, pela sua diversidade e ocupação de papéis ecológicos, muitos dos quais, chave nas comunidades, representa um dos elementos de maior eficácia para indicar as condições ambientais, auxiliando na identificação de processos de degradação e na criação e implantação de medidas conservacionistas (Gonzaga 1986).

No intuito de preservar um remanescente importante da Mata Atlântica do sul da Bahia, foi criada em 1998, em uma área de 85.686 ha, a Área de Proteção Ambiental do Pratigi (APA do Pratigi), que abrange cinco municípios (Ibirapitanga, Igrapiúna, Ituberá, Nilo Peçanha e Piraí do Norte). As investigações sobre a avifauna da Mata Atlântica do baixo sul da Bahia, ainda são incipientes e concentradas, sobretudo, na costa do cacau (Cordeiro 2000, Cordeiro 2003, Laps et al 2003, Silveira 2005). Esta situação é totalmente insatisfatória diante de uma região com rica biodiversidade de fauna e flora e que vem sofrendo, historicamente, grandes agressões e transformações.

Coleções ornitológicas sempre foram centros de produção e difusão do conhecimento básico sobre a diversidade e distribuição de aves no Brasil congregando dados acerca da avifauna de uma região, sua biogeografia e ecologia e estudos de anatomia, taxonomia e sistemática molecular, importantes para subsidiar políticas conservacionistas de ambientes e espécies, além da descrição de novos táxons (Vuilleumier 1998; Aleixo e Straub 2007). A Divisão de Aves do Museu de Zoologia da Universidade Estadual de Feira de Santana (DAMZFS), lotada no Laboratório de Ornitologia/UEFS, foi criada em 2005 (Deliberação CGEN no 121 de 05/08/2005; DOU 01/09/2005) e, além de conter espécimes taxidermizadas, também conta com as coleções de tecidos (amostra de material genético) e de carcaças com vísceras dos espécimes tombados, conservadas em álcool $70 \%$.

A análise da dieta das aves constitui uma importante ferramenta para a compreensão da ecologia de uma comunidade. Conhecer quais e em que quantidades ou proporções esses elementos compõem a dieta de uma determinada espécie é a premissa básica para o entendimento das relações tróficas entre as espécies (Piratelli e Pereira 2002). A investigação da dieta em aves começou na década de $40 \mathrm{com}$ a análise do conteúdo estomacal (Moojen et al 1941), que foi uma das primeiras metodologias para este estudo.

Apesar de ser um dos grupos mais bem conhecidos, em muitos aspectos os estudos sobre as aves ainda são escassos. A maioria das informações sobre a ecologia alimentar das aves que se tem registro é de estudos a partir de observações em campo, que principalmente no caso de aves insetívoras, é pouco preciso. Estudos de dieta permitem melhor entendimento da história natural da espécie e fornece base para o desenvolvimento de estratégias de conservação (Gonçalves et al. 2007). Pipridae (rendeiras, tangarás e afins) e Thraupidae (saíras, tiês, sanhaços e afins) são famílias de aves passeriformes estritamente americanas e conhecidas por terem diversas espécies dispersoras de sementes, a despeito de também se alimentarem de insetos (Sick 1997). No entanto, há variação da proporção de uso destes itens 
alimentares entre suas diferentes espécies, podendo haver alterações de acordo com o ambiente e sua sazonalidade (Sick 1997). O presente estudo objetivou analisar o conteúdo estomacal de espécimes das famílias Pipridae e Thraupidae do acervo da DA/MZFS, provenientes da Área de Proteção Ambiental do Pratigi (APA do Pratigi).

\section{MATERIAL E MÉTODOS}

O material biológico usado no trabalho foi o estômago de espécimes testemunhos que foram coletados na APA do Pratigi, baixo sul da Bahia, e encontravam-se armazenados na DA/MZFS. A coleta das aves foi feita em duas áreas distintas da APA do Pratigi, Ecopolo I e Ecopolo II, com auxílio de redes ornitológicas e sob licença permanente para coleta de aves SISBIO 13192-1, entre os anos de 2012 e 2014. A análise do conteúdo estomacal foi restrita a indivíduos das famílias Pipridae e Thraupidae.

As aves estudadas foram fixadas com formol $4 \%$, injetado na cavidade abdominal e torácica, bem como no interior do tubo digestivo logo após a coleta e preparação das peles. As carcaças dos animais permaneceram nessa solução por alguns dias e em seguida foram transferidas para álcool etílico $70 \%$. Os espécimes foram separados e com o auxílio de um bisturi, foi aberta a cavidade torácica através de uma incisão feita no lado esquerdo do peito. Rebateu-se, então, a porção aberta do peito, para que se pudesse retirar o trato gástrico do indivíduo. Foi feito um corte na porção basal do esôfago e outro na parte distal do reto (bem próximo ao lado interno da cloaca). Feito isso, foram transferidos para frascos individualizados, contendo álcool etílico $70 \%$, e identificados com etiqueta correspondente. Uma vez retirado o trato digestivo, o mesmo foi aberto e todo o conteúdo foi triado e identificado, sendo anotados o número de itens encontrados e parte do corpo (no caso de insetos), tudo foi devidamente fotografado.

O conteúdo estomacal foi triado em placa de Petri com álcool 70\%, e analisado com o auxílio de lupa, e seus itens identificados quando possível. A identificação foi baseada em bibliografias específicas (Borror e DeLong 1988).

\section{RESULTADOS E/OU DISCUSSÃO}

Foram analisados os estômagos de 30 espécimes, distribuídos em 16 espécies de aves, sendo cinco da família Pipridae e 11 da família Thraupidae. Dois tipos de recursos foram encontrados nas análises: vegetais e artrópodes; deste último tipo foram encontrados 58 itens (Tabela 1). Dez espécies tiveram dieta constituída tanto por insetos como por material vegetal, cinco espécies por apenas material vegetal e uma espécie apenas por inseto (Tabela 1).

Dentre os artrópodes encontrados nas amostras, predominaram indivíduos das ordens Coleoptera $(32,7 \%)$ e Hymenoptera $(20,6 \%)$ (Figura 1); nesta última prevaleceu a família Formicidae $(66,7 \%)$. Os principais consumidores de formigas foram Volatinia jacarina e Manacus manacus.

Verificou-se no presente estudo, assim como citado em literatura, que as aves da família Pipridae são prioritariamente frugívoras; cabe ressaltar que foi observada a presença de insetos em quatro das cinco espécies analisadas. Mesmo com uma dieta estritamente frugívora, aves necessitam de proteína animal para a formação e desenvolvimento dos ovos e para a alimentação dos ninhegos, garantindo seu crescimento. Assim, é usual que supram esta necessidade de proteína animal se alimentando de artrópodes, recurso abundante em florestas tropicais (Sick 1997).

Cabe destacar que padrões sazonais influenciam na abundância de frutos e artrópodes, que são as principais fontes de alimentos de aves de sub-bosque. Os picos sazonais observados na distribuição espacial de artrópodes estão relacionados com o período 
reprodutivo da maioria dos passeriformes (Develey e Peres 2000). Este fato é corroborado pelo trabalho realizado por Riehl e Adelson (2008), ao observar a dieta de frugívoros durante seu período reprodutivo, verificando consumo de artrópodes por adultos e filhotes. A disponibilidade sazonal de alimentos também influencia a composição, riqueza e abundância da comunidade de aves (Tebbich et al. 2004).

Tabela 1: Contribuição relativa na composição alimentar de algumas aves das famílias Pipridae e Thraupidae, provenientes da Área de Proteção Ambiental do Pratigi (APA do Pratigi), no estado da Bahia. Col $=$ Coleoptera, Hem = Hemiptera, Hym = Hymenoptera, For $=$ Formicidae, Ara $=$ Araneae, Lep $=$ Lepdoptera, Iso $=$ Isoptera, Ovo $=$ Ovos de Hemiptera, Mat. Veg. $=$ Material vegetal, $\mathrm{Sem}=$ sementes, $\mathrm{Veg}=$ material vegetal não determiado, Gra $=$ grão de areia. Os números entre parênteses referem-se ao número de indivíduos analisados. Material vegetal, foi configurado como a presença da polpa e pele do fruto, pois é difícil de mensurar. $\mathrm{X}=$ presença.

\begin{tabular}{|c|c|c|c|c|c|c|c|c|c|c|c|c|}
\hline Família & Espécie & $\mathrm{Col}$ & $\mathrm{Hem}$ & Hym & For & Ara & Lep & Iso & Ovo & Sem & Veg & Gra \\
\hline \multirow{5}{*}{ Pipridae } & Ceratopipra rubrocapilla (4) & & 1 & & & & & & 9 & 3 & $\mathrm{X}$ & \\
\hline & Chiroxiphia pareola (2) & & & & & & & & & 4 & $\mathrm{X}$ & \\
\hline & Dixiphia pipra (2) & & & & & & & & & 28 & & \\
\hline & Machaeropterus regulus (3) & 3 & & & & & & & & 21 & $\mathrm{X}$ & \\
\hline & Manacus manacus (2) & & & & 2 & & & & & 13 & $\mathrm{X}$ & \\
\hline \multirow[t]{11}{*}{ Thraupidae } & Chlorophanes spiza (1) & 5 & & 4 & & & 1 & & & & $\mathrm{X}$ & \\
\hline & Coereba flaveola (1) & & 6 & & & & & & & & & \\
\hline & Ramphocelus bresilius (2) & 1 & 1 & & & 1 & & & & & $\mathrm{X}$ & \\
\hline & Sporophila leucoptera (2) & 3 & & & & & & 5 & & & $\mathrm{X}$ & \\
\hline & Tachyphonus rufus (1) & 6 & & & 1 & & & & & 17 & & $\mathrm{X}$ \\
\hline & Tangara cyanomelas (1) & & & & & & & & & 1 & $\mathrm{X}$ & \\
\hline & Tangara palmarum (2) & & & & & 1 & & & & 4 & $\mathrm{X}$ & \\
\hline & Tangara sayaca (2) & & & & & & & & & 2 & $\mathrm{X}$ & \\
\hline & Tangara seledon (3) & 1 & & & 1 & 2 & & & & 45 & $\mathrm{X}$ & \\
\hline & Tiaris fuliginosus (1) & & & & & & & & & 13 & $\mathrm{X}$ & $\mathrm{X}$ \\
\hline & Volatinia jacarina (1) & & & & 4 & & & & & & $\mathrm{X}$ & $\mathrm{X}$ \\
\hline Total & & 19 & 8 & 4 & 8 & 4 & 1 & 5 & 9 & 152 & & \\
\hline
\end{tabular}

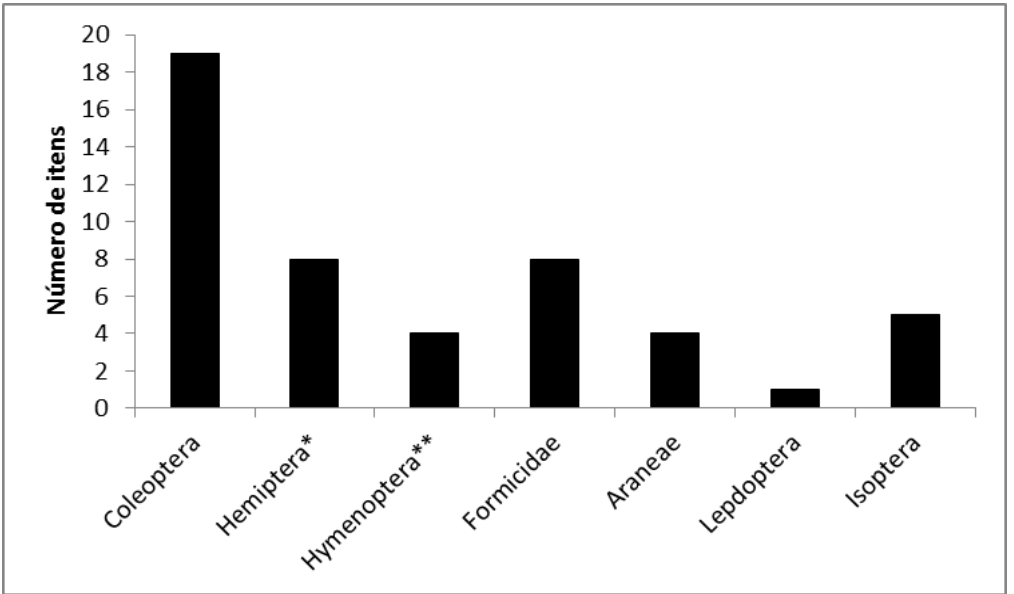

Figura 1: Proporção de grupos de artrópodes nas espécies de aves da Família Pipridae e Thraupidae provenientes da Área de Proteção Ambiental do Pratigi, Bahia, depositada na coleção da Divisão de Aves do Museu de Zoologia da Universidade Estadual de Feira de Santana. (* Exceto os ovos; ** Exceto Formicidae).

\section{CONCLUSÃO}

Estudos que visam conhecer a dieta das aves são importantes, pois fornecem dados sobre a estrutura trófica da comunidade de aves e condições ambientais, colaborando para estabelecimento de estratégias de conservação. Para um conhecimento mais a fundo e uma 
melhor definição dos grupos tróficos das espécies analisadas, se faz necessário uma avaliação com uma maior quantidade de indivíduos, levando em consideração que algumas espécies só tiveram um único espécime analisado.

Os resultados alcançados no presente estudo demonstram que foi possível implantar e iniciar, junto ao Laboratório de Ornitologia, uma nova linha de pesquisa em dieta de aves e aplicando multidisciplinaridade uma vez que abrange também outras áreas de conhecimento, como a entolomogia. Apesar das análises terem sido restritas a algumas espécies, pode se conceber que os resultados positivos sirvam de base para a implantação futura de um serviço regular de análise de conteúdo estomacal de aves.

\section{REFERÊNCIAS}

ALEIXO, A. e F. C. Straub. 2007. Coleções de aves brasileiras: breve histórico diagnóstico atual e perspectivas para o futuro. Revista Brasileira de Ornitologia, 15(2):315-324.

BORROR, D. J e D. M. DeLong. 1988. Introdução ao estudo dos insetos. São Paulo: Edgard Blucher. 653p ISBN (Broch.).

COMITÊ BRASILEIRO DE REGISTROS ORNITOLÓGICOS. 2014. Lista Primária das aves do Brasil. Disponível em <http://www.cbro.org.br/CBRO/listabr.htm>.

CORDEIRO, P.H.C. 2000. Estudo da avifauna em fragmentos da Mata Atlântica do Sul da Bahia (levantamento de espécies). Relatório Técnico. [s.l: s.n.].

2003. A fragmentação da Mata Atlântica no sul da Bahia e suas implicações na conservação dos psitacídeos. Em: Prado, P.I., E. C. Landau, R. T. Moura, L. P. S. Pinto, G. A. B. Fonseca e K. N. Alger (Orgs.). Corredor de Biodiversidade da Mata Atlântica do Sul da Bahia. Ilhéus: IESB/CI/CABS/UFMG/UNICAMP, Disponível em: $<$ http://ibama.angelfire.com/papagaios.pdf $>$.

DEVELEY, P. F. e C. A. Peres. 2000. Resource seasonality and the structure of mixed species birds flocks in a coastal Atlantic Forest of southeastern Brazil. Journal of Tropical Ecology 16: 33 - 53.

GONÇALVES, R. M. M., M. P. Teixeira, K. M. Caldeira, C. A. Lima, M. A. Z. Borges e L. O. Leite. 2007. Análise do Conteúdo estomacal de seis famílias de passeriformes (Aves). Anais do VIII Congresso de Ecologia do Brasil - Caxambu- Minas Gerais.

GONZAGA, L. P. 1986. Composição da avifauna em uma parcela de mata perturbada na baixada, em Majé, Estado do Rio de Janeiro, Brasil. Dissertação de Mestrado. Rio de Janeiro: Universidade Federal do Rio de Janeiro. Biblioteca do Museu Nacional.

LAPS, R. R., P. H. C. Cordeiro, D. Kijawa, R. Ribon, A. A. F. Rodrigues e A. Uejima. 2003. Aves. Em: Rambaldi, D. M. e D. A. S. Oliveira (orgs.). Fragmentação de Ecossistemas. Ministério do Meio Ambiente, Brasília, DF.

MOOJEN, J., J. C. Carvalho, H. S. Lopes. 1941. Observações sobre conteúdo gástrico das aves brasileiras. Memórias do Instituto Osvaldo Cruz, 36:405-444.

PIRATELLI, A., M. R. Pereira. 2002. Dieta das aves na região leste de Mato Grosso do Sul, Brasil. Ararajuba, 10 (2): 131-139.

RIEHL, C. e G. S. Adelson. 2008. Seasonal insectivory by Black-headed Trogons, a tropical dry forest frugivore. Journal Field Ornithology 79: 371 - 380

Sick, H. 1997. Ornitologia Brasileira. Ed. Nova Fronteira, Rio de Janeiro.

SILVEIRA, L.F., P. F. Develey, J. F. Pacheco and B. M. Whitney. 2005. Avifauna of the Serra das Lontras-Javi montane complex, Bahia, Brazil. Cotinga, 24: 45-54.

TEBBICH, S.; M. Tabordky; B. Fessl; M. Dvorak; H. Windler. 2004. Feeding behavior of four arboreal Darwin's finches: adaptations to spatial and seasonal variability. The Condor 106: 95-105.

VUILLEUMIER, F. 1998. The need to colected birds in Neotopics. Ornitologia Neotropical, 9:201-203. 